\title{
Cyclical coronary flow reductions in conscious dogs equipped with ameroid constrictors to produce severe coronary narrowing
}

\author{
K. P. Gallagher, G. Osakada, W. S. Kemper, and J. Ross, Jr. \\ From the Seaweed Canyon Laboratory, Division of Cardiology, Department of Medicine, \\ University of California, San Diego, La Jolla, California (U.S.A.)
}

\begin{abstract}
Summary
In conscious dogs equipped with ameroid constrictors to produce gradual coronary occlusion, coronary flow velocity was monitored prior to complete occlusion when coronary constriction was severe (resting flow velocity reduced by $10-50 \%$ from control recordings made $7-10$ days after ameroid implantation). In six of the ten dogs, we observed spontaneous cyclical variations in coronary flow velocity, characterized by gradual reduction in flow followed by very abrupt restoration of flow. The cyclic coronary flow reductions were observed between 20 and 31 days after ameroid implantation. These changes in flow bear striking similarity to those observed by previous investigators using anesthetized, open-chest canine preparations, in which the role of platelets was clearly demonstrated. Consequently, we hypothesize that spontaneous platelet aggregation and de-aggregation within the severely narrowed coronary lumen (enclosed by the ameroid constrictors) could account for our observations.
\end{abstract}

Key words: ameroid constriction, conscious dogs, coronary stenosis, cyclical coronary flow reductions, coronary flow velocity

\section{Introduction}

This report describes our initial observations of spontaneous cyclical coronary blood flow variations in conscious dogs equipped with ameroid constrictors to produce gradual coronary occlusion. Cyclical reductions in coronary blood flow velocity were observed in dogs with severely narrowed vessels, which bear a striking resemblance to coronary flow changes originally reported by Folts et al. (4). These investigators, using an open chest, anesthetized canine model, constricted coronary arteries with plastic rings to produce narrowing of 60-80\% diameter reduction and documented spontaneous changes in coronary blood flow characterized by gradual reductions in coronary flow followed by abrupt increases (4). The cyclic coronary flow alterations were attributed to gradual accumulation and sudden loss of platelet plugs from the narrowed coronary lumen, a conclusion supported by several subsequent studies $(1,2,5,9)$.

We wanted to determine if cyclical flow changes in stenosed coronary arteries might also be observed in conscious animals equipped with ameroid constrictors. We were further interested in the mechanism by which ameroid constrictors eventually stop coronary blood flow, since we have never observed a totally obliterated coronary lumen produced by

Supported by Specialized Center of Research on Ischemic Heart Disease HL-17682 from the National Heart, Lung, and Blood Institute 
ameroid constriction, rather severely narrowed arteries which contained adherent blood clots which completely occluded the vessel. We hypothesized that cyclic accumulation and loss of platelet aggregates, as suggested by Folts et al. (4) and Aiken et al. (1), may precede the totally occlusive thrombus formation.

\section{Methods}

Nine mongrel dogs were instrumented during sterile surgery with a continuous wave Doppler flowprobe on the circumflex coronary artery to measure coronary blood flow velocity, and distal to the flowprobe an ameroid ring (with internal diameter of $3.5 \mathrm{~mm}$ ) was placed around the vessel as previously described (6). Onc additional dog was instrumented with a pulsed Doppler flowprobe and ameroid constrictor (with $2.5 \mathrm{~mm}$ diameter). Ameroid is a casein material with hygroscopic properties that cause it to swell in the presence of fluid (11). The ameroid rings were in slotted metal sleeves, which allowed the swelling ameroid material to encroach only upon the enclosed vessel. Gradual coronary constriction occurs with collateral development in the dependent areas, the usual time to complete coronary occlusion being approximately three to four weeks $(3,6)$.

Initial evaluations of coronary blood flow velocity at rest were performed 7 to 10 days postoperatively. Then, between 2 and 4 weeks postoperatively, the dogs were returned to the laboratory and coronary flow velocity was monitored at least 15 minutes while the dogs lay quietly on a rug on the floor. When cyclical changes in flow velocity occurred, they were usually evident immediately and at that point longer periods of recording were initiated (60-240 minutes). Blood flow velocity was recorded in the nine dogs with continuous wave Doppler probes by telemetry, as we have previously described (6), and was calibrated in frequency of zero-cross detections in kilohertz $(\mathrm{kHz})$ by demodulating the directly recorded audio signal through a zero-cross detector. The reliability of coronary blood flow velocity as a relative indicator of volume flow changes by this technique has been previously demonstrated (10). In the dog with a pulsed Doppler flowprobe, coronary flow velocity signals were processed with a flowmeter constructed in this laboratory. At the time of necropsy, gross examination of the blood vessels at the location of the flowprobe and ameroid occluder revealed localized thickening of the vessel wall and severe concentric narrowing (within the ameroid occluder). There were no obvious differences in this pattern among the ten animals included in the study.

\section{Results}

Of the ten dogs evaluated for cyclical coronary flow velocity changes prior to ameroid induced occlusion, six demonstrated spontaneous reductions. Recordings where made in this group of dogs between 14 and 31 days postoperatively and cyclic flow variations were evident between 20 and 31 days. The other four dogs were evaluated at a similar interval after implantation of the ameroid constrictors, but no cyclical flow variations were observed. The reduction in mean coronary flow velocity (compared with recordings made 7 to 10 days postoperatively) varied from $10-50 \%$ whether cyclical flow variations were observed or not, indicating severe partial obstruction. An example of slow speed recordings from one of the six dogs exhibiting the general features of the cyclical flow variations is presented in figure 1 . Each cycle is characterized by a gradual downslope of decreasing flow velocity ended by a very abrupt increase in flow velocity. There was considerable variability in the frequency and size of the cyclical changes but, when detected, they were consistently observed for periods of 60 minutes or longer (fig. 1). The dogs were frequently asleep, as was the case in the example shown in figure 1 . Spontaneous physical activity could be readily observed and distinguished from spontaneous cyclical flow reductions because flow velocity increased without a preceding interval of gradual and progressive flow velocity reduction. In addition, comments on the activity level of the dogs were made continuously on the voice channel of the tape which was also used to directly record the audio flow velocity signal. 

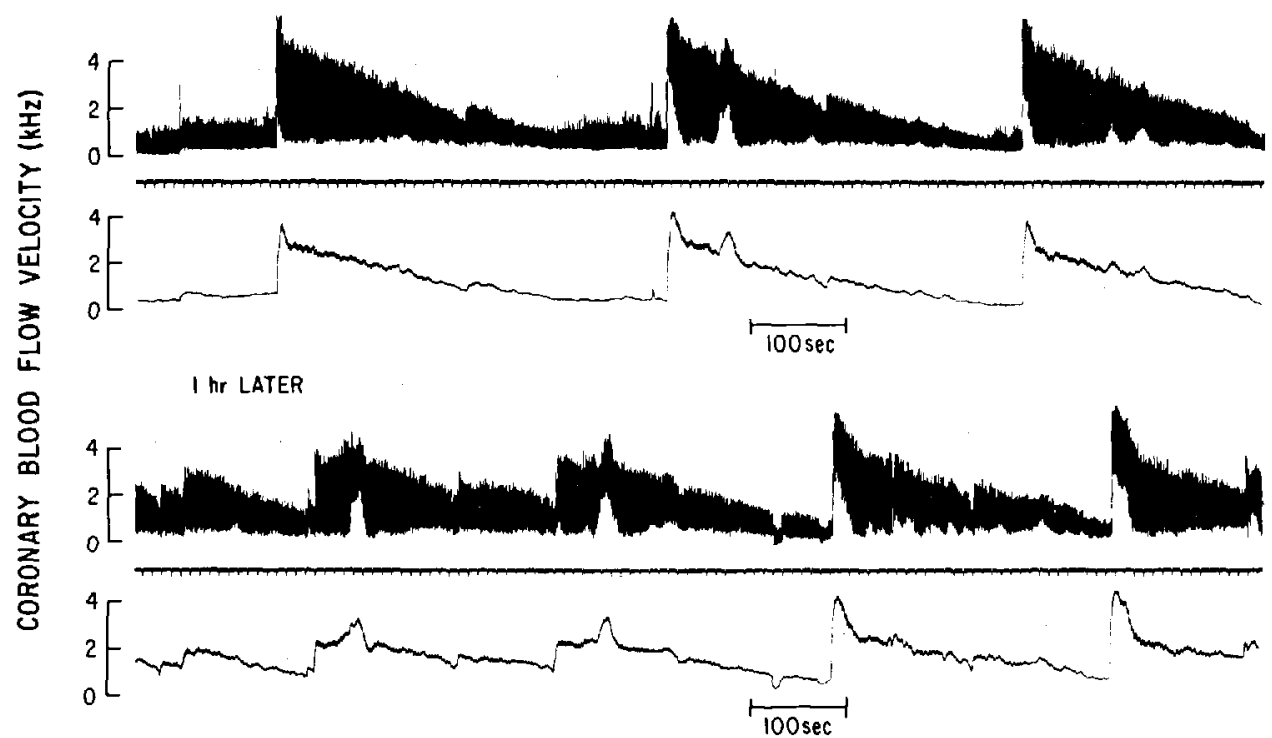

Fig. 1. Example of coronary blood flow velocity tracings at slow paper speed to illustrate cyclical coronary flow variations which persisted, relatively uniformly, for an entire two hour recording period. The upper tracing shows phasic flow velocity tracings, while the lower tracing shows mean flow velocity. The direct audio signal from the continuous wave Doppler flowmeter was recorded on magnetic tape and later played back through a zero-cross detector which was used to demodulate the flow signal and generate the wave forms shown here. In the upper portion of the figure, recordings were obtained while the dog slept. In the lower portion, approximately one hour later, the dog was awake but remained lying down, occasionally lifting its head to look around.

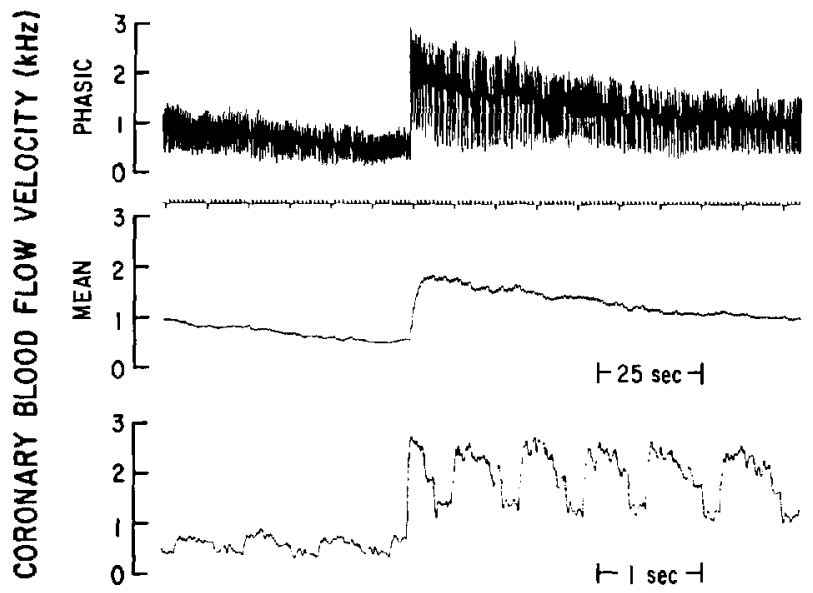

Fig. 2. Recordings of abrupt flow velocity rise at faster paper speed than in figure 1 , to illustrate the rapidity with which the changes in flow velocity occurred. In the lower panel are shown expanded recordings, at higher paper speed, of the cardiac cycles just before and after the rapid flow change, including the exact cycle during which the increase occurred. Flow velocity increased spontaneously at mid diastole in this dog who was lying down, apparently asleep. Heart rate remained quite stable throughout this episode. 
The abruptness of flow change is demonstrated in figure 2 , an example of one of the flow variations in another dog. In the upper two panels, phasic and mean tracings of coronary flow velocity are shown at low paper speed. The lower panel in figure 2, at higher paper speed, shows the exact cardiac cycle during which the abrupt flow change occurred. During mid-diastole the flow velocity nearly tripled indicating a very rapid change in resistance. This change was unaccompanied by any physical activity, as confirmed by remarks on the voice track of the tape and this dog also appeared to be asleep. The phasic pattern of flow velocity prior to the increase is characteristic of severe coronary stenosis, with reduced diastolic relative to systolic flow. Note the absence of change in heart rate.

In five of the six dogs exhibiting cyclic flow variations we gave one or two aspirin tablets ( 300 or $600 \mathrm{mg}$ ) orally. An example of recordings from one of these dogs, given one tablet of aspirin ( $300 \mathrm{mg}$ ), is shown in figure 3. Approximately 75 minutes later, substantial attenuation of flow variations was evident. A similar effect was observed in one other dog administered $300 \mathrm{mg}$ of aspirin. In an additional two dogs, aspirin exerted little or no effect; an example of recordings from one of these dogs is shown in figure 4 . There appeared to be a diminution in the amplitude of the cyclical flow variations 30 minutes after $300 \mathrm{mg}$ aspirin (orally), but within 15 minutes variations comparable to the pre-aspirin condition were

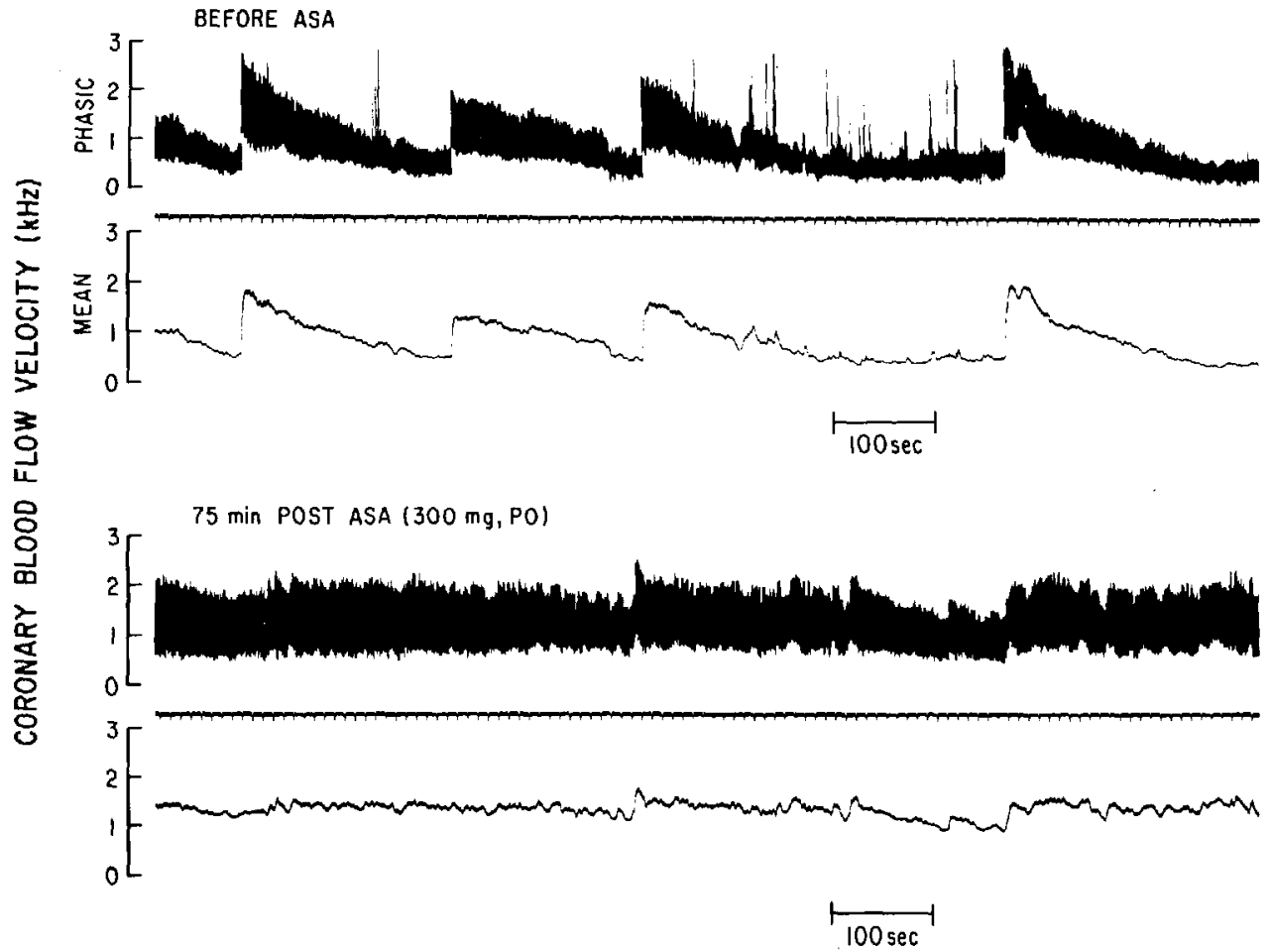

Fig. 3. Slow paper speed recording from one of the dogs before (upper) and after (lower) oral administration of one aspirin tablet $(300 \mathrm{mg})$. Cyclic flow variations of high amplitude are evident before aspirin, but 75 minutes after aspirin relatively small changes in flow velocity were observed. A similar effect was observed on one other dog given $300 \mathrm{mg}$ aspirin. The spikes in the phasic flow trace (upper portion) are artifact due to interference with the telemetry link between the Doppler flowmeter transmitter and receiver. 


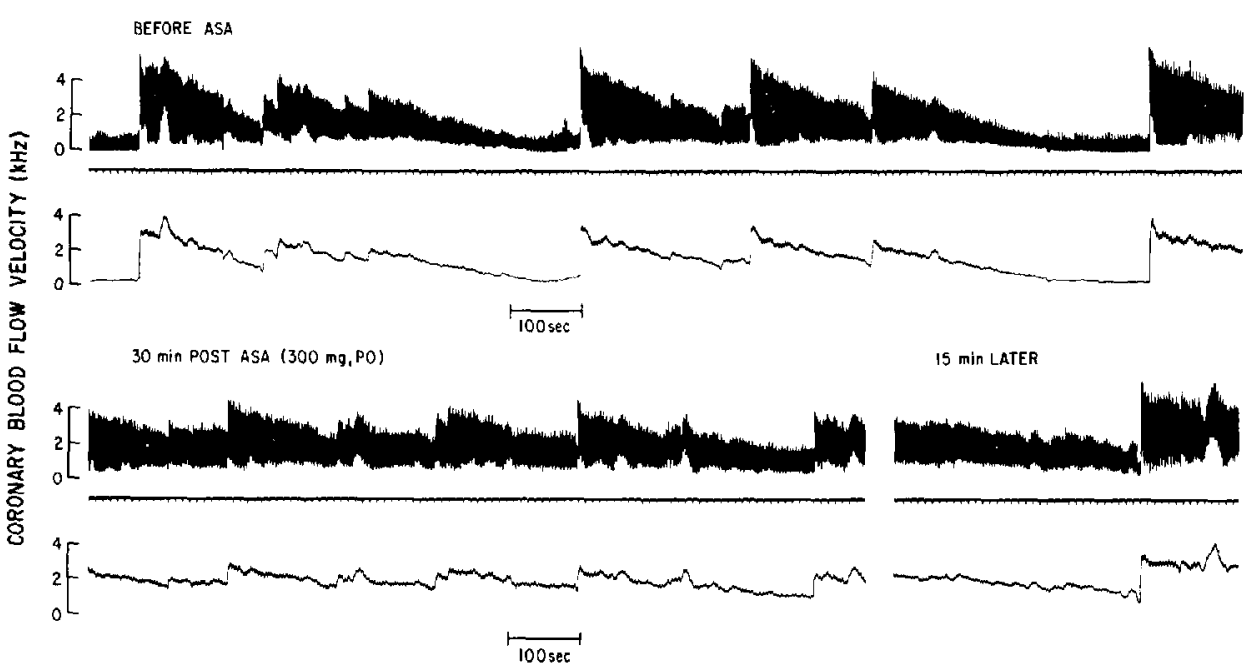

Fig. 4. Slow paper speed recording from a different dog, also given aspirin ( $300 \mathrm{mg}$ ). Thirty minutes after aspirin, there appears to be some attenuation in the amplitude of the cyclic flow variations. The effect, if any, of the aspirin was short-lived, because 15 minutes later (lower portion, right end) cyclical flow variations comparable to the pre-aspirin recordings were observed. A similar pattern was observed in one other dog. Thus, aspirin appeared to limit coronary flow variations in two dogs, but failed to do so in another two dogs. In a fifth dog, aspirin was given after coronary flow appeared to shut down completely. Flow was restored gradually two hours later, but whether or not aspirin caused this change is conjectural.

observed which persisted after an additional $300 \mathrm{mg}$ of aspirin were administered. In the fifth dog, after observing five cyclic flow reductions, it appeared that the coronary artery was permanently occluded in that flow was not restored after the last cycle. This dog was given one tablet $(300 \mathrm{mg}$ ) of aspirin anyway and returned to its cage. Two hours later recordings of flow velocity were reinitiated, at which time coronary flow steadily increased over a period of approximately one hour. No further cycles were observed although the coronary artery was permanently occluded five days later.

\section{Discussion}

The literature suggests that platelets may be responsible for the cyclical flow variations observed in our dogs with ameroid constrictors. The primary evidence supporting this view are the flow velocity tracings themselves and their similarity to those recorded by Folts et al. $(4,5)$, and Aiken $(1,2)$, together with setting of the observations, that is, a severely narrowed coronary artery. The flow variations we observed are also similar to changes documented by Patterson et al. (8) in conscious dogs equipped with intracoronary electrodes designed to produce progressive coronary thrombosis, which lends additional support to our hypothesis. These investigators demonstrated gradual coronary flow reduction, interrupted spontaneously by abrupt increases in flow which gradually diminished in size as stenosis severity increased over a matter of hours.

We have not seen anything comparable to the gradual reduction followed by an abrupt (within one cardiac cycle) increase of flow in any other condition. We hypothesize that 
spasm was not the primary event in our observations because of the gradual slope of flow reduction (rather than abrupt closure) followed by extremely rapid increase in flow. This pattern seems to be inconsistent with the concept of spasm, characterized by fast onset and gradual recovery as documented by cineangiography of coronary vessels during vasospastic episodes (7). These lines of evidence, although compelling, are indirect, and confirmation that our observations correspond to those recorded previously will require additional studies, particularly with regard to platelet function tests and the role of coronary spasm as an alternative explanation for the observations. In addition, our dogs were studied only at rest or while they were asleep. The effect of different levels of activity, high versus low flow velocity, relative degree of vagal versus sympathetic influences, and variable perfusion pressure on the phenomenon of cyclical flow reductions remain to be determined.

Folts et al. (5) recently reported that dogs with chronically implanted plastic rings (with fixed internal diameter) exhibit cyclical flow variations, but it was necessary to maintain these dogs on aspirin and two of the seven dogs died. The ameroid preparations provides a different type of chronic animal model for study of flow variations, because gradual occlusion allows time for collateral development which means that cyclical reductions in coronary inflow will be less likely to jeopardize survival of the animal. On the other hand, the time available for study is limited to the interval available between severe narrowing and complete closure of the vessel. In addition, we observed cyclical flow variations in only six of ten dogs, whereas all seven dogs equipped with plastic cylinders by Folts et al. (5) showed changes. Whether our findings reflect actual absence of flow variations in some of our dogs, or the possibility that we monitored flow velocity in these dogs at the wrong time we cannot say with certainty.

In conclusion, our findings in conscious dogs provide indirect support for the view that spontaneous platelet plugging may cyclically aggravate coronary artery stenosis. These observations are preliminary and caution is necessary in their interpretation, but they are striking and warrant further investigation. If these variations are due to platelets, it lends credibility to the hypothesis that spontaneous platelet aggregates can form and break free in chronically narrowed vessels in conscious animals. The ameroid-equipped conscious dog may provide a suitable model for further characterization of cyclical coronary flow variation, elucidation of the mechanisms responsible for this phenomenon, and because of extensive collateral development, allow evaluation of interventions with less risk of sudden death.

\section{Acknowledgement}

We would like to thank Michael D. McKown and Daniel P. McKown for the technical assistance that made it possible to do this study.

\section{References}

1. Aiken JW, Gorman RR, Shebuski RJ (1979) Prevention of blockage of partially obstructed coronary arteries with prostacyclin correlates with inhibition of platelet aggregation. Prostaglandins 17:483-494

2. Aiken JW, Shebuski RJ (1980) Comparison in anesthetized dogs of the anti-aggregatory and hemodynamic effects of prostacyclin and a chemically stable prostacyclin analog, 6a-CARBA-PGI2 (carbocyclin). Prostaglandins 19:629-643

3. Elliot EC, Jones EL, Bloor CM, Leon AS, Gregg DE (1968) Day-to-day changes in coronary hemodynamics secondary to constriction of circumflex branch of left coronary artery in conscious dogs. Circ Res 22:237-250

4. Folts JD, Crowell ED, Rowe GG (1976) Platelet aggregation in partially obstructed vessels and its elimination with aspirin. Circulation 54:365-370 
5. Folts JD, Gallagher KP, Rowe GG (1982) Blood flow reductions in stenosed canine coronary arteries: Vasospasm or platelet aggregation? Circulation 65:248-255

6. Kumada T, Gallagher KP, Shirato K, McKown D, Miller M, Kemper WS, White F, Ross J Jr (1980) Reduction of exercise-induced regional myocardial dysfunction by propranolol. Studies in a canine model of chronic coronary artery stenosis. Circ Res 46:190-200

7. Maseri A, Severi S, DeNes M, L'Abbate, Chiechia S, Marzilli M, Ballestra AM, Parodi O, Giagini A, Distant A (1978) "Variant" angina: One aspect of a continuous spectrum of vasospastic myocardial ischemia. Am J Cardiol 42:1019-1035

8. Patterson E, Holland K, Eller BT, Lucchesi BR (1982) Ventricular fibrillation resulting from ischemia at a site remote from previous myocardial infarction. A conscious canine model for sudden coronary death. Am J Cardiol 50:1414-1423

9. Shebuski RJ, Aiken JW (1980) Angiotension II-induced renal prostacyclin release suppresses platelet aggregation in the anesthetized dog. In: Samuelsson B, Ramwell PW, Pooletti P. (eds), Advances in prostaglandin and thromboxane research, vol. 7 Raven Press, New York, p 1149-1152

10. Vatner SF, Franklin D, VanCitters RL (1970) Simultaneous comparison and calibration of the Doppler and electromagnetic flowmeters. J Appl Physiol 29:907-910

11. Vineberg A, Mahanti B, Litvak J (1960) Experimental gradual coronary artery constriction by ameroid constrictors. Surg 47:765-771

Received April 12, 1984

Authors' address:

Kim P. Gallagher, Ph.D., Thoracic Surgery Research Laboratory, R3484 Kresge I, Box 056, University of Michigan, Ann Arbor, Michigan 48109 (U.S.A.)

Für die Schriftleitung verantwortlich: Prof. Dr. R. Jacob, Physiologisches Institut II, Gmelinstraße 5, D-7400 Tübingen, und Prof. Dr. W. Schaper, Institut für experimentelle Kardiologie in William-G.-Kerckhoff-Institut, Benekestraße 2, D-6350 Bad Nauheim. - Anzeigenverwaltung: Springer-Verlag GmbH \& Co. KG, Kurfürstendamm 237, D-1000 Berlin 15 - Verantwortlich für den Anzeigenteil: E. Lückermann. - Verlag: Dr. Dietrich Steinkopff Verlag GmbH \& Co. KG, Saalbaustraße 12, Postfach 111008 , D-6100 Darmstadt 11. - Satz und Druck: Ungeheuer + Ulmer KG GmbH + Co, Körnerstraße 14-18, Postfach 1040, D-7140 Ludwigsburg. 